\title{
Perceived Exertion is Not Necessarily Associated with Altered Brain Activity during Exercise
}

\author{
Kenichi Shibuya $^{1)}$, Chihoko Ueda ${ }^{1)}$, Kohei Sato ${ }^{1)}$, Shizuyo Shimizu-Okuyama ${ }^{2)}$, Mitsuru Saito ${ }^{3)}$, \\ Atsuko Kagaya $^{1)}$, Mifuyu Kamo ${ }^{4)}$, Takuya Osada ${ }^{5)}$ and Tomoko Sadamoto ${ }^{1)}$ \\ 1) Research Institute of Physical Fitness, Japan Women's College of Physical Education \\ 2) Institute of Physical Education, Keio University \\ 3) Applied Physiology Laboratory, Toyota Technological Institute \\ 4) Laboratory of Exercise Physiology, Faculty of Physical Education, Japan Women's College of Physical Education \\ 5) Department of Sports Medicine for Health Promotion, Tokyo Medical University
}

\begin{abstract}
Previous studies have investigated the relationship between prefrontal cortex activation and perceived exertion during prolonged exercise. However, the effect of perceived exertion on prefrontal cortex activity is confounded by exercise intensity. Therefore, the changes in prefrontal cortex activity in response to perceived exertion remain unclear. The purpose of the present study was to investigate the relationship between the activation (oxygenation) of the prefrontal cortex and perceived exertion during constant work-rate elbow-flexion exercise with or without muscle-spindle stimulation. Ten healthy, right-handed subjects participated in the study. Nearinfrared spectroscopy with probes positioned over the prefrontal cortex measured its activation throughout elbowflexion exercise. Subjects performed sustained elbow-flexion exercise at $25-35 \%$ of the maximal voluntary contraction (MVC) with or without muscle-spindle stimulation (vibration), which can decrease perceived exertion. The ratings of perceived exertion were significantly lower during exercise with vibration $(\mathrm{Ex}-\mathrm{Vib})$ than during exercise without vibration (Ex) $(p<0.05)$. The oxygenation of the prefrontal cortex during Ex-Vib did not significantly differ from that during Ex $(p>0.05)$. These results indicated that perceived exertion was not necessarily associated with prefrontal cortex activation during exercise. J Physiol Anthropol 28(2): 63-69, 2009 http://www.jstage.jst.go.jp/browse/jpa2
\end{abstract}

[DOI: $10.2114 / j p a 2.28 .63]$

Keywords: motor task, perceived exertion, prefrontal cortex, near-infrared spectroscopy

\section{Introduction}

Humans have been fascinated by prolonged exercise performance since antiquity, and numerous physiological and psychological experiments have addressed what limits endurance, i.e., what causes fatigue. Physiological fatigue is characterized by the exercise-induced loss of the power- and force-generating ability of a muscle during the course of or after exercise (Bigland-Ritchie and Woods, 1984; Booth and Thomason, 1991; Nybo and Nielsen, 2001; Gandevia, 2001).

Fatigue may be considered an increased difficulty in maintaining a given exercise intensity, as assessed using ratings of perceived exertion (RPEs), e.g., the Borg scale (Borg, 1975, 1982), which are used for the quantification of the perception of effort (Williamson et al., 2001; Nybo and Nielsen, 2001; Dalsgaard et al., 2002; Nybo, 2003). This method of fatigue evaluation is based on a subjective sensation rather than on an objective assessment of the changes in force or power, and it does not enable a clear discrimination between the peripheral and central factors contributing to fatigue.

Previous studies have investigated the relationship between brain activation and the sensation of fatigue, as assessed using RPEs (Dalsgaard and Secher, 2007; Dalsgaard et al., 2002, Dalsgaard et al., 2003, Dalsgaard et al., 2004a, Dalsgaard et al., 2004b, Dalsgaard et al., 2004c; González-Alonso et al., 2004; Nybo et al., 2003; Nybo and Nielsen, 2001). In particular, Nybo and Nielsen (2001) reported a significant correlation between prefrontal cortex activity and RPEs during cycling exercise. However, as stated in their study, the significant relationship between RPEs and prefrontal cortex activation was influenced by not only the sensation of fatigue but also the effect of exercise intensity.

Cerebral oxygenation changes reflect cerebral functional activation (Colier et al., 1997; Colier et al., 1999; Kleinschmidt et al., 1996; Shibuya et al., 2004a, 2004b; Shibuya and Tachi, 2006; Shibuya and Kuboyama, 2007; Shibuya et al., 2008; Obrig et al., 1996), and these changes can be measured using several techniques. Near-infrared spectroscopy (NIRS) and functional magnetic resonance imaging enable the noninvasive 
monitoring of regional changes in cortical tissue oxygenation in response to various stimuli (Colier et al., 1999; Kleinschmidt et al., 1996; Mehagnoul-Schipper et al., 2000; Obrig et al., 1996, Obrig et al., 2000).

NIRS permits the monitoring of changes in the oxy$\left(\left[\mathrm{HbO}_{2}\right]\right)$, deoxy- $([\mathrm{Hb}])$, and total hemoglobin $([\mathrm{tHb}])$ levels with high temporal resolution. The NIRS method is based on changes in absorption that depend on changes in the concentrations of $\left[\mathrm{HbO}_{2}\right]$ and $[\mathrm{Hb}]$ in the tissue under investigation.

In fact, Ide et al. (1999) and Ide and Secher (2000) reported that prefrontal cortex activation (oxygenation) increased with increase in exercise intensity. To avoid the interfusion of the effects of exercise intensity and perceived exertion on prefrontal activation, the experimental design should set the exercise intensity to a stable level. The muscle-spindle stimulation technique (vibration) assists or opposes an exercising muscle group while developing a given force. The vibration technique gives the changes in the perceived exertion at the same internal- and external-workloads (Goodwin et al., 1972), and it helps in understanding the effects of the changes in perceived exertion on prefrontal cortex activity. This technique has been commonly used in previous studies (e.g., Ogoh et al., 2002; Goodwin et al., 1972). The purpose of the present study was to determine whether perceived exertion was associated with prefrontal cortex activity by using the musclespindle stimulation technique during static exercise.

\section{Methods}

\section{Subjects and paradigm}

Ten normal, right-handed females (age, $21.0 \pm 0.7$ years; height, $159.9 \pm 4.8 \mathrm{~cm}$; and weight, $55.6 \pm 5.9 \mathrm{~kg}$ ) participated in the present study. Informed consent was obtained from each subject after providing a detailed explanation of the procedure and the noninvasiveness of the study. The criterion for the inclusion of subjects was a medical history free of cardiovascular, pulmonary, renal, endocrinological, and neurological disorders. The subjects were instructed not to train strenuously on the day prior to testing and not to exercise on the day of testing. Moreover, they were asked to refrain from consuming food or beverages containing caffeine before the test. All subjects provided informed, written consent for the procedures.

\section{Muscle-spindle stimulation (vibration)}

A vibrator was used to induce muscle contraction by the reflex stimulation of the distal tendon of the biceps brachii. In the present study, the oscillating vibration was applied to the distal tendon of the biceps brachii, while the subjects performed sustained isometric contractions of the biceps brachii. The oscillating frequency of the vibrator was $100 \mathrm{~Hz}$, and its amplitude was $0.8 \mathrm{~mm}$. Vibration of the distal tendon of the biceps brachii during elbow flexion aided tension development in the biceps brachii muscle. In the resting condition, $7.7 \% \pm 3.2 \%$ of the maximal voluntary contraction (MVC) was achieved by the application of vibration.

\section{Exercise protocol}

Throughout the study, the subjects sat on a comfortable chair in a quiet room. Each subject performed 2 sessions of elbow-flexion exercise with or without muscle-spindle stimulation (Ex-Vib and Ex respectively). The 2 exercise sessions were separated by a 20-min rest. The order of these sessions was randomized. The RPE (the subject rated her perceived effort on the Borg scale (Borg, 1975)) was recorded immediately after the exercise.

On the first day, in order to determine the MVC, the subjects performed maximal static elbow flexion at an angle of $90^{\circ}$ (biceps brachii muscle exercise) with their dominant arm (right arm) for a total of 3 times. After the MVC trials, the subject was familiarized with the experimental protocol and the physiological measurements to be obtained.

On the second day, the subjects performed static elbow flexion at a $90^{\circ}$ angle. After a preliminary 30-min resting period in the upright position, each subject performed exercises under the same protocol under 2 different conditions: (i) elbow flexion (Ex) and (ii) elbow flexion with stimulation of the distal tendon of the biceps brachii (Ex-Vib). The protocol consisted of a 3-min resting control period followed by a 2-min static contraction at $30 \%$ of the subject's pre-determined MVC. All exercise bouts were separated by a rest period of a minimum of $20 \mathrm{~min}$.

\section{Near-infrared Spectroscopy (NIRS)}

Prefrontal tissue oxygenation levels were recorded from the muscle and brain by using an NIRS unit (Hamamatsu NIRO200; Hamamatsu Photonics KK, Tokyo, Japan). The theory and specifics of NIRS have been described in detail elsewhere (Elwell et al., 1994). In brief, near-infrared light is transmitted via a fiber-optic cable and passed into the tissue of interest through an emitting optode. Any light that is transmitted through the tissue is acquired by a detector optode and carried by a second fiber-optic cable to a photon detector (photomultiplier tube) in the spectrometer. The NIRO-200 uses laser diodes set at 4 different wavelengths $(776,826,845$, and $905 \mathrm{~nm}$ ) to provide the light-source signal, which is pulsed in rapid succession and detected by the photomultiplier tube. The probes were bilaterally positioned over the motor cortex areas enclosing F3, according to the modified international electroencephalography (EEG) 10-20 system (American Electroencephalographic Society, 1994), as described in previous studies (Shibuya et al., 2004a, 2004b, 2006; Bhambhani et al., 2007). An interoptode spacing of $4 \mathrm{~cm}$ was used to separate the emitting from the detecting sensor. The optodes were held within a black rubber housing that maintained constant optode spacing. The housing was taped to the skin and covered with an optically dense vinyl sheet that was also affixed with tape. The housing and vinyl sheet minimized the loss of near-infrared light from the field of 
interrogation as well as the intrusion of light from the environment. The full assembly was further secured with an elastic bandage that encircled the forearm and head. The levels of $\left[\mathrm{HbO}_{2}\right],[\mathrm{Hb}]$, and $[\mathrm{tHb}]$ were continuously sampled by the NIRS unit at a rate of $2 \mathrm{~Hz}$. These parameters are expressed (in $\left.\mathrm{mM}^{*} \mathrm{~cm}\right)$ as a change from 0 . The values of $\left[\mathrm{HbO}_{2}\right]$ and $[\mathrm{Hb}]$ during exercise were calculated using the average of 10 datasets $(5 \mathrm{~s})$.

\section{Pulmonary gas exchange}

The subjects breathed through a face mask connected to a hot wire flowmeter that measured respiratory flow and was calibrated using a $3-\mathrm{L}$ syringe. A small sample $(1 \mathrm{~mL} / \mathrm{s})$ of expired gas was withdrawn continuously from the mask and analyzed for $\mathrm{O}_{2}$ and $\mathrm{CO}_{2}$ by using a mass spectrometer (Arco2000, Arco Inc., Japan). The mass spectrometer was calibrated with fresh air and precision gas $\left(\mathrm{O}_{2}, 15.04 \%\right.$ and $\mathrm{CO}_{2}$, $4.957 \%)$. The time delay between the flow and gasconcentration signals was calculated to obtain breath-by-breath data. Pulmonary gas-exchange data were collected every $5 \mathrm{~s}$. The heart rate (HR) was measured using leads and an electrocardiogram monitor (OEC-6201; Nihon-Kohden, Japan).

\section{Cardiopulmonary measurements}

Mean arterial pressure (MAP) was measured continuously using a photoplethysmometer (Finometer; Finapres Medical Systems, Arnhem, The Netherlands) with the cuff on the middle finger of the nondominant hand.

\section{Statistical analysis}

To compare the time-course changes, two-way ANOVA was applied to the parameters in the Ex and Ex-Vib conditions, using time and condition (with or without vibration) as fixed factors. To assess the time-course change in each condition, the changes in the parameters were subjected to post-hoc analysis using the Dunnet test. A $p$ value less than 0.05 was considered statistically significant.

\section{Results}

The mean MVC was $14.1 \pm 2.2 \mathrm{kp}$. The perceived exertions

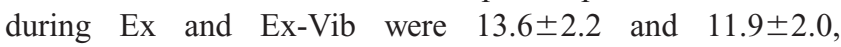
respectively, and the difference between them was significant $(t=4.636, p<0.01)$. The RPEs immediately after the exercise also significantly differed $(t=4.636, p<0.05)$; they were $13.6 \pm 2.2$ in the Ex condition and 11.9 \pm 2.0 in the Ex-Vib condition.

\section{$H R, M A P$, and end-tidal $\mathrm{CO}_{2}$}

ANOVA revealed a significant change in the HR with time and no significant changes between the Ex and Ex-Vib conditions (time: $\mathrm{F}=32.642, p<0.01 ; \mathrm{Ex}$ vs. Ex-Vib: $\mathrm{F}=0.279, p>0.05$; and interaction: $\mathrm{F}=0.388, p>0.05)$, and the post-hoc tests revealed that the values from $15 \mathrm{~s}$ after the start of exercise to the end of exercise were significantly higher than those at the baseline $(p<0.05)$ under both the Ex and Ex-Vib conditions. Furthermore, the MAP also significantly differed with time and not with the presence or absence of vibration (time: $\mathrm{F}=49.742, p<0.01$; Ex vs. Ex-Vib: $\mathrm{F}=1.0100, p>0.05$; and interaction: $\mathrm{F}=0.601, p>0.05$ ), and the post-hoc test for MAP showed that the values from the start of exercise to the end of exercise were significantly higher than those at the baseline $(p<0.05)$ under both the Ex and Ex-Vib conditions (Table 1).

The end-tidal $\mathrm{CO}_{2}\left(\mathrm{ETCO}_{2}\right)$ slightly increased, but the change was not significant; further, it did not significantly differ between the Ex and Ex-Vib conditions (time: $\mathrm{F}=0.818$, $p>0.05$; Ex vs. Ex-Vib: $\mathrm{F}=0.360, p>0.05$; and interaction: $\mathrm{F}=0.608, p>0.05)($ Table 1$)$.

\section{Prefrontal cortex oxygenation}

With regard to $\left[\mathrm{HbO}_{2}\right]$, [Hb], and [tHb] levels, ANOVA revealed significant changes with time and no significant changes between the Ex and Ex-Vib conditions. The results of the statistical tests for these levels are as follows: (i) $\left[\mathrm{HbO}_{2}\right]$ : time, $\mathrm{F}=57.397$ and $p<0.01$; Ex vs. Ex-Vib, $\mathrm{F}=0.031$ and $p>0.05$; and interaction, $\mathrm{F}=0.189$ and $p>0.05$ (Fig. 1). (ii) $[\mathrm{Hb}]$ : time, $\mathrm{F}=75.781$ and $p<0.01$; Ex vs. Ex-Vib, $\mathrm{F}=0.0004$

Table $1 \mathrm{ETCO}_{2}$, $\mathrm{HR}$ and MAP changes in Ex and Ex-Vib conditions

\begin{tabular}{llll}
\hline & & Baseline value & $\begin{array}{l}\text { At the end } \\
\text { of exercise }\end{array}$ \\
\hline ETCO $_{2}(\%)$ & Ex & $3.71 \pm 1.0$ & $3.93 \pm 1.0$ \\
& Ex-Vib & $3.84 \pm 1.1$ & $4.06 \pm 1.0$ \\
$\mathrm{HR}(\mathrm{bpm})$ & Ex & $72.5 \pm 3.5$ & $87.0 \pm 8.8^{*}$ \\
& Ex-Vib & $72.2 \pm 5.7$ & $85.5 \pm 8.4^{*}$ \\
$\mathrm{MAP}(\mathrm{mmHg})$ & Ex & $80.7 \pm 7.6$ & $94.6 \pm 11.1^{*}$ \\
& Ex-Vib & $79.8 \pm 7.3$ & $98.4 \pm 6.7^{*}$ \\
\hline
\end{tabular}

Astariscs shows significant difference from baseline values $(p<0.05)$.

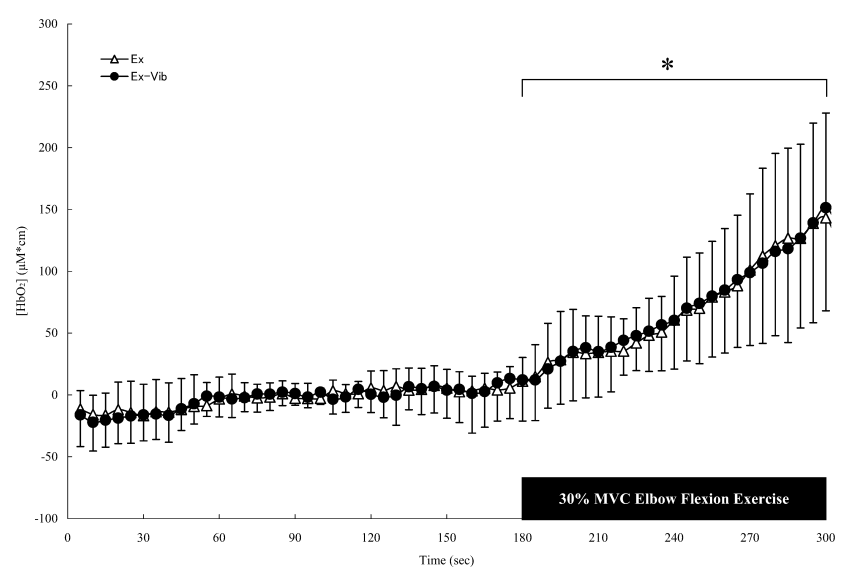

Fig. 1 Oxyhemoglobin $\left(\left[\mathrm{HbO}_{2}\right]\right)$ levels in the prefrontal cortex. Changes in the baseline values of $\left[\mathrm{HbO}_{2}\right]$ concentration in the prefrontal cortex during exercise without (Ex) or with muscle spindle stimulation (ExVib). Values are represented as means \pm SD. The asterisks show significant differences from the baseline values, $p<0.05$. 


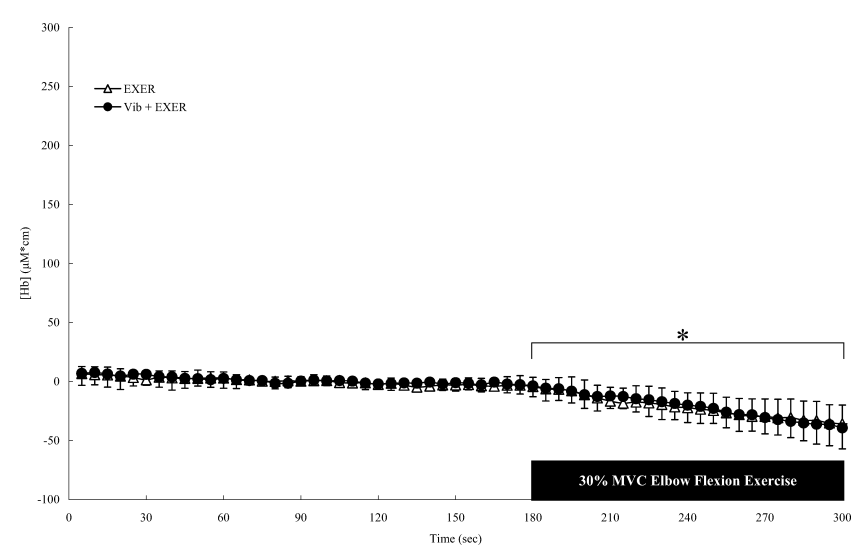

Fig. 2 Deoxyhemoglobin ([Hb]) levels in the prefrontal cortex. Changes in the baseline values of $[\mathrm{Hb}]$ concentration in the prefrontal cortex during exercise without (Ex) or with muscle spindle stimulation (ExVib). Values are represented means \pm SD. The asterisks show significant differences from the baseline values, $p<0.05$.

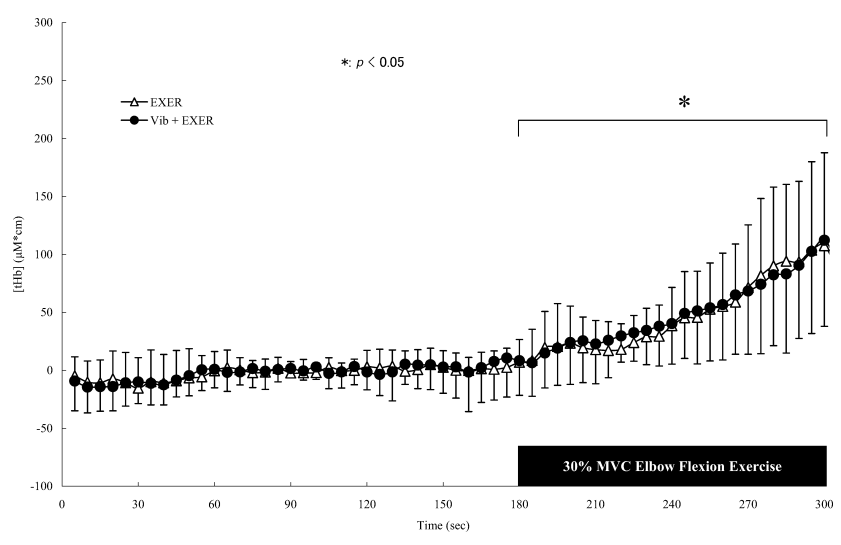

Fig. 3 Total hemoglobin ([tHb]) levels in the prefrontal cortex. Changes in the baseline values of [tHb] concentration in the prefrontal cortex during exercise without (Ex) or with muscle spindle stimulation (ExVib). Values are represented means \pm SD. The asterisks show significant differences from the baseline values, $p<0.05$.

and $p>0.05$; and interaction, $\mathrm{F}=0.4587$ and $p>0.05$ ) (Fig. 2). (iii) $[\mathrm{tHb}]$ : time, $\mathrm{F}=57.394$ and $p<0.01$; Ex vs. Ex-Vib, $\mathrm{F}=0.031$ and $p>0.05$; and interaction, $\mathrm{F}=0.189$ and $p>0.05$ ) (Fig. 3).

The post-hoc test for changes in the $\left[\mathrm{HbO}_{2}\right],[\mathrm{Hb}]$, and $[\mathrm{tHb}]$ levels under the Ex and Ex-Vib conditions revealed that the values from the start of exercise to the end of exercise were significantly higher than those at the baseline $(p<0.05)$.

\section{Discussion}

The present study revealed no significant difference in the prefrontal cortex activity during exercise under the Ex and ExVib conditions.

Borg's RPEs have enabled the measurement of impending fatigue during exercise testing (Borg, 1982; Ekblom and Goldbarg, 1971). The RPE increases with increase in exercise intensity (Ceci and Hassman, 1991; Dunbar et al., 1992; Eston et al., 1987; Glass et al., 1992; Kurokawa and Ueda, 1992; Ueda et al., 2006). Additionally, Nybo and Nielsen (2001) found a significant correlation between the RPEs and prefrontal cortex activity. The prefrontal cortex is most likely involved in the initiation of volitional movements (Nowak et al., 1999; Pedersen et al., 1998). The activity (oxygenation) of the prefrontal cortex increases with increase in exercise intensity (Ide et al., 1999). As such, the prefrontal cortex activity described by Nybo and Nielsen (2001) is a reflection of 2 dimensions, i.e., exercise intensity and the RPE. Thus, in order to determine the relationship between the RPE and prefrontal cortex activity, the effect of the RPE should be separated from that of exercise intensity.

The exercise design in the present study differed from that in a previous study (Nybo and Nielsen, 2001), and it successfully separated the effects of RPE from those of exercise intensity by using the muscle-spindle stimulation technique. We detected no significant difference in the activity of the prefrontal cortex in the Ex and Ex-Vib conditions. This result indicates that the RPEs are not associated with prefrontal cortex activity. The RPEs reflect the sensation of physical fatigue (Borg, 1982; Ekblom and Goldbarg, 1971). Information regarding physical fatigue might be transmitted from the peripheral nervous system to the sensory cortex and not to the prefrontal cortex. As mentioned by Ueda et al. (2006), the RPE scale might be influenced by multiple psychological factors since perceived exertion is induced by central pain and several peripheral factors, namely, arm pain, leg pain, breathing difficulty, and heart pain. On the other hand, the activity of the prefrontal cortex might not reflect multiple factors. In the present study, we proposed the linear increase in the oxygenation in the prefrontal cortex during steady-state exercise (Figs. 1, 2, 3). The reasons for the increase in the oxygenation remain unclear in the present study. Further studies are needed to clarify the reasons for the increase in prefrontal cortex oxygenation even during steady-state exercise.

In the present study, no significant differences were detected in the central circulation parameters (i.e., HR and MAP) in the Ex and Ex-Vib conditions. Additionally, the $\mathrm{ETCO}_{2}$ did not vary from the baseline value in both conditions. $\mathrm{ETCO}_{2}$ is a key factor for the regulation of cerebral blood volume (Harris et al., 2006). Thus, this indicated that cerebral blood volume was not substantially altered. The significant increase in the prefrontal cortex activity in both conditions suggests that cerebral autoregulation occurred in the present study.

We used NIRS to investigate human brain activation. NIRS can noninvasively measure cerebral hemoglobin oxygenation (Jobsis, 1977) and has been used to assess functional brain activation in adults (Chance et al., 1993; Hoshi and Tamura 1993; Villringer et al., 1993) and infants (Taga et al., 2003; Taga and Asakawa, 2007). NIRS signals reflect the fact that blood flow response to functional activation is larger than the increase in oxygen consumption, similar to the blood oxygenation level-dependent (BOLD) contrast in fMRI 
(Villringer and Change, 1997). The oxygenation response typically consists of an increase in oxygenated hemoglobin (oxy-Hb) and a slight decrease in deoxygenated hemoglobin (deoxy- $\mathrm{Hb}$ ) over an activated cortical region (Obrig and Villringer, 2003). Because NIRS imposes fewer body constraints, the technique has been used to investigate brain activation in relation to motor function (Colier et al., 1999; Franceschini et al., 2003; Hatakenaka et al., 2007; Hirth et al., 1996; 1997; Hupper et al., 2006; Jasdzewski et al., 2003; Klinschmit et al., 1996; Maki et al., 1995; Obrig et al., 1996; Sato et al., 2005, 2006; Shimada et al., 2004; Watanabe et al., 1996; Wolf et al., 2002). It should be noted that the Hb signals reflect the products of the molar concentration changes and the optical path lengths. The optical path lengths may vary between brain regions. Since we performed the group analysis on a channel-by-channel basis, the statistical results were not affected by the regional-dependent differences of the optical path lengths. Although the optical path lengths may vary between participants, consistent changes in the $\mathrm{Hb}$ signals are good approximations of those in the $\mathrm{Hb}$ concentrations that are induced by brain activation.

In conclusion, the present study revealed that oxygenation of the prefrontal cortex did not significantly differ in the Ex and Ex-Vib conditions. The results of the present study suggest that perceived exertion is not necessarily associated with prefrontal cortex activity.

Acknowledgement We thank Ms. Ai Hirasawa for her expert technical help. The study was supported by the "Academic Frontier" Project for Private Universities (Japan Women's College of Physical Education): matching fund subsidy from the Ministry of Education, Culture, Sports, Science and Technology of Japan.

\section{References}

American Electroencephalographic Society (1994) Guideline thirteen: guidelines for standard electrode position nomenclature. J Clin Neurophysiol 11: 111-113

Bhambhani Y, Malik R, Mookerjee S (2007) Cerebral oxygenation declines at exercise intensities above the respiratory compensation threshold. Respir Physiol Neurobiol 156: 196-202

Bigland-Ritchie B, Woods JJ (1984) Changes in muscle contractile properties and neural control during human muscular fatigue. Muscle Nerve 7: 691-699

Booth FW, Thomason DB (1991) Molecular and cellular adaptation of muscle in response to exercise: perspectives of various models. Physiol Rev 71: 541-585

Borg G (1975) Simple rating for estimation of perceived exertion. In Borg G ed., Physical work and effort. Pergamon Press, New York, 39-46

Borg GA (1982) Psychophysical bases of perceived exertion. Med Sci Sports Exerc 14: 377-381

Ceci R, Hassman P (1991) Self-mentioned exercise at three different RPE-intensities in treadmill vs field running. Med Sci Sports Exerc 23: 732-738

Chance B, Zhuang Z, UnAh C, Alter C, Lipton L (1993) Cognition-activated low-frequency modulation of light absorption in human brain. Proc Natl Acad Sci USA 90: 3770-3774

Colier WN, Binkhorst RA, Hopman MT, Oeseburg B (1997) Cerebral and circulatory haemodynamics before vasovagal syncope induced by orthostatic stress. Clin Physiol 17: 83-94

Colier WN, Quaresima V, Oeseburg B, Ferrari M (1999) Human motor-cortex oxygenation changes induced by cyclic coupled movements of hand and foot. Exp Brain Res 129: 457-461

Dalsgaard MK, Ide K, Cai Y, Quistorff B, Secher NH (2002) The intent to exercise influences the cerebral O2/carbohydrate uptake ratio in humans. J Physiol 540: 681-689

Dalsgaard MK, Nybo L, Cai Y, Secher NH (2003) Cerebral metabolism is influenced by muscle ischaemia during exercise in humans. Exp Physiol 88: 297-302

Dalsgaard MK, Ogoh S, Dawson EA, Yoshiga CC, Quistorff B, Secher NH (2004a) Cerebral carbohydrate cost of physical exertion in humans. Am J Physiol Regul Integr Comp Physiol 287: R534-540

Dalsgaard MK, Quistorff B, Danielsen ER, Selmer C, Vogelsang T, Secher NH (2004b) A reduced cerebral metabolic ratio in exercise reflects metabolism and not accumulation of lactate within the human brain. J Physiol 554: $571-578$

Dalsgaard MK, Secher NH (2007) The brain at work: a cerebral metabolic manifestation of central fatigue? J Neurosci Res 85: 3334-3339

Dalsgaard MK, Volianitis S, Yoshiga CC, Dawson EA, Secher NH (2004c) Cerebral metabolism during upper and lower body exercise. J Appl Physiol 97: 1733-1739

Dunbar CC, Robertson RJ, Baun R, Blandin MF, Metz K, Burdett R, Goss FL (1992) The validity of regulating exercise intensity by ratings of perceived exertion. Med Sci Sports Exerc 24: 94-99

Elwell CE, Cope M, Edwards AD, Wyatt JS, Delpy DT, Reynolds EO (1994) Quantification of adult cerebral hemodynamics by near-infrared spectroscopy. J Appl Physiol 77: 2753-2760

Ekblom B, Goldbarg AN (1971) The influence of physical training and other factors on the subjective rating of perceived exertion. Acta Physiol Scand 83: 399-406

Eston RG, Davies BL, Williams JG (1987) Use of perceived effort ratings to control exercise intensity in young healthy adults. Eur J Appl Physiol Occup Physiol 56: 222-224

Franceschini MA, Fantini S, Thompson JH, Culver JP, Boas DA (2003) Hemodynamics evoked response of the sensorimotor cortex measured noninvasively with nearinfrared optical imaging. Psychophysiology 40: 548-560

Gandevia SC (2001) Spinal and supraspinal factors in human 
muscle fatigue. Physiol Rev 81: 1725-1789

Glass SC, Knowlton RG, Becque MD (1992) Accuracy of RPE form graded exercise training intensity. Med Sci Sports Exerc 24: 1303-1307

González-Alonso J, Dalsgaard MK, Osada T, Volianitis S, Dawson EA, Yoshiga CC, Secher NH (2004) Brain and central haemodynamics and oxygenation during maximal exercise in humans. J Physiol 557: 331-342

Goodwin GM, McCloskey DI, Mitchell JH (1972) Cardiovascular and respiratory responses to changes in central command during isometric exercise at constant muscle tension. J Physiol 226: 173-190

Harris AD, Ide K, Poulin MJ, Frayne R (2006) Control of endtidal $\mathrm{PCO} 2$ reduces middle cerebral artery blood velocity variability: implications for physiological neuroimaging. Neuroimage 29: 1272-1277

Hatakenaka M, Miyai I, Mihara M, Sakoda S, Kubota K (2007) Frontal regions involved in learning of motor skilla functional NIRS study. Neuroimage 34: 109-116

Hirth C, Obrig $\mathrm{H}$, Villringer $\mathrm{K}$, Thiel A, Bernarding J, Muhlenickel W, Flor H, Dirnagl U, Villinger A (1996) Noninvasive functional mapping of the human motor cortex using near-infrared spectroscopy. Neuroreport 7: 1977-1981

Hoshi Y, Tamura M (1993) Detection of dynamic changes in cerebral oxygenation coupled to neuronal function during mental work in man. Neurosci Lett 150: 5-8

Huppert TJ, Hoge RD, Diamond SG, Franceschini MA, Boas DA (2006) A temporal comparison of BOLD, ASL, and NIRS hemodynamic responses to motor stimuli in adult humans. Neuroimage 29: 368-382

Ide K, Horn A, Secher NH (1999) Cerebral metabolic response to submaximal exercise. J Appl Physiol 87: 1604-1608

Ide K, Secher NH (2000) Cerebral blood flow and metabolism during exercise. Prog Neurobiol 61: 397-414

Jasdzewski G, Strangman G., Wagner J, Kwong KK, Poldrack PA, Boas DA (2003) Differences in the hemodynamic response to event-related motor and visual paradigms as measured by near-infrared spectroscopy. Neuroimage 20: 479-488

Jobsis FF (1977) Noninvasive, infrared monitoring of cerebral and myocardial oxygen sufficiency and circulatory parameters. Science 198: 1264-1267

Kleinschmidt A, Obrig H, Requardt M, Merboldt KD, Dirnagl U, Villringer A, Frahm J (1996) Simultaneous recording of cerebral blood oxygenation changes during human brain activation by magnetic resonance imaging and near-infrared spectroscopy. J Cereb Blood Flow Metab 16: 817-826

Kurokawa T, Ueda T (1992) Validity of ratings of perceived exertion as an index of exercise intensity in swimming training. Ann Physiol Anthrop 11: 277-288

Maki A, Yamashita Y, Ito Y, Watanabe E, Mayanagi Y, Koizumi H (1995) Spatial and temporal analysis of human motor activity using noninvasive NIR topography. Med Phys 22: 1997-2005

Mehagnoul-Schipper DJ, Vloet LC, Colier WN, Hoefnagels
WH, Jansen RW (2000) Cerebral oxygenation declines in healthy elderly subjects in response to assuming the upright position. Stroke 31: 1615-1620

Nowak M, Olsen KS, Law I, Holm S, Paulson OB, Secher NH (1999) Command-related distribution of regional cerebral blood flow during attempted handgrip. J Appl Physiol 86: 819-824

Nybo L, Nielsen B (2001) Perceived exertion is associated with an altered brain activity during exercise with progressive hyperthermia. J Appl Physiol 91: 2017-2023

Obrig H, Hirth C, Junge-Hülsing JG, Döge C, Wolf T, Dirnagl U, Villringer A (1996) Cerebral oxygenation changes in response to motor stimulation. J Appl Physiol 81: $1174-1183$

Obrig H, Neufang M, Wenzel R, Kohl M, Steinbrink J, Einhäupl K, Villringer A (2000) Spontaneous low frequency oscillations of cerebral hemodynamics and metabolism in human adults. Neuroimage 12: 623-639

Obrig H, Villringer A (2003) Beyond the visible-imaging the human brain with light. J Cereb Blood Flow Metab 23: 1-18

Ogoh S, Wasmund WL, Keller DM, O-Yurvati A, Gallagher KM, Mitchell JH, Raven PB (2002) Role of central command in carotid baroreflex resetting in humans during static exercise. J Physiol 543: 349-364

Pedersen JR, Johannsen P, Bak CK, Kofoed B, Saermark K, Gjedde A (1998) Origin of human motor readiness field linked to left middle frontal gyrus by MEG and PET. Neuroimage 8: 214-220

Roick H, Vongiesen HJ, Benecke R (1993) On the origin of the postexcitatory inhibition seen after transcranial magnetic brain stimulation in awake human subjects. Exp Brain Res 151: $330-337$

Sato H, Fuchino Y, Kiguchi M, Katura T, Maki A, Yoro T, Koizumi H (2005) Intersubject variability of near-infrared spectroscopy signals during sensorimotor cortex activation. J Biomed Opt 10: 044001

Sato H, Kiguchi M, Maki A, Fuchino Y, Obata A, Yoro T, Koizumi H (2006) Within-subject reproducibility of nearinfrared spectroscopy signals in sensorimotor activation. J Biomed Opt 11: 014021

Shibuya K, Kuboyama N (2007) Human motor cortex oxygenation during exhaustive pinching task. Brain Res 1156: $120-124$

Shibuya K, Tanaka J, Kuboyama N, Ogaki T (2004a) Cerebral oxygenation during intermittent supramaximal exercise. Respir Physiol Neurobiol 140: 165-172

Shibuya K, Tanaka J, Kuboyama N, Murai S, Ogaki T (2004b) Cerebral cortex activity during supramaximal exhaustive exercise. J Sports Med Phys Fitness 44: 215-219

Shibuya K, Tachi M (2006) Oxygenation in the motor cortex during exhaustive pinching exercise. Respr Physiol Neurobiol 153: 261-266

Shibuya K, Sadamoto T, Sato K, Moriyama M, Iwadate M (2008) Quantification of delayed oxygenation in ipsilateral primary motor cortex compared with contralateral side 
during a unimanual dominant-hand motor task using nearinfrared spectroscopy. Brain Res 1210: 142-147

Shimada S, Hiraki K, Matsuda G, Oda I (2004) Decrease in prefrontal hemoglobin oxygenation during tasks with delayed visual feedback: a near-infrared spectroscopy study. Cogn Brain Res 20: 480-490

Taga G, Asakawa K (2007) Selectivity and localization of cortical response to auditory and visual stimulation in awake infants aged 2 to 4 months. Neuroimage 36: 1246-1252

Taga G, Asakawa K, Maki A, Konishi Y, Koizumi H (2003) Brain imaging in awake infants by near-infrared optical topography. Proc Natl Acad Sci USA 1000: 10722-10727

Ueda T, Nabetani T, Teramoto K (2006) Differential perceived exertion measured using a new visual analogue scale during pedaling and running. J Physiol Anthropol 25: 171-177

Villringer A, Chance B (1997) Non-invasive optical spectroscopy and imaging of human brain function. Trends Neurosci 20: 435-442

Villringer A, Planck J, Hock C, Schleinkofer L, Dirnagle U (1993) Near infrared spectroscopy (NIRS): a new tool to study hemodynamic changes during activation of brain function in human adults. Neurosci Lett 154: 101-104

Watanabe E, Yamashita Y, Maki A, Ito Y, Koizumi H (1996)
Non-invasive functional mapping with multi-channel near infra-red spectroscopic topography in humans. Neurosci Lett 205: 41-44

Williamson JW, McColl R, Mathews D, Mitchell JH, Raven PB, Morgan WP (2001) Hypnotic manipulation of effort sense during dynamic exercise: cardiovascular responses and brain activation. J Appl Physiol 90: 1392-1399

Wolf M, Wolf U, Toronov V, Michalos A, Paunescu A, Choi JH, Gratton E (2002) Different time evolution of oxyhemoglobin and deoxyhemoglobin concentration changes in the visual and motor cortices during functional stimulation: a near-infrared spectroscopy study. Neuroimage 16: 704-712

Received: October 8, 2008

Accepted: December 25, 2008

Correspondence to: Kenichi Shibuya, Research Institute of Physical Fitness, Japan Women's College of Physical Education, 8-19-1 Kitakarasuyama, Setagaya-ku, Tokyo 157-8565, Japan

Phone: +81-3-3300-6175

Fax: +82-3-3307-5825

e-mail: kshibuya@jwcpe.ac.jp 